\title{
LITERATURVERZEICHNIS
}

Für Primärliteratur wird im Folgenden in eckigen Klammern ein Normtitel angegeben, der aus einer Abkürzung und einem in der Regel lateinischen Volltitel besteht. Die Abkürzung entspricht so weit wie möglich der Abkürzung bei Lampe. Als vollständiger Titel wird, wo vorhanden, die Titelform der CPG benutzt, sonst eine eigene Formulierung. (Dabei werden gewisse Uneinheitlichkeiten, etwa die differierende Schreibung i- / j-, in Kauf genommen.) Sekundärliteratur wird mit einem kursiv geschriebenen Kürzel bezeichnet, in der Regel aus dem Namen und einem Wort des Titels bestehend. Die alphabetische Reihenfolge im Literaturverzeichnis orientiert sich bei biblischen Büchern nach der Folge in der Bibel, bei der übrigen Primärliteratur an der Abkürzung, bei der Sekundärliteratur am Kürzel.

\section{Biblische Bücher}

Biblische Stellenangaben werden grundsätzlich nach einer der folgenden Editionen zitiert:

[Editio Gottingensis] Septuaginta. Vetus Testamentum Graecum. Auctoritate Academiae Scientiarum Gottingensis editum, Göttingen 1974-2014

[Septuaginta ed. Rahlfs/Hanhart] Septuaginta, Id est Vetus Testamentum graece iuxta LXX interpretes, edidit Rahlfs, Alfred. Editio altera, quam recognovit et emendavit Hanhart, Robert. Duo volumina in uno, Stuttgart 2006

[Novum Testamentum ed. Nestle/Aland/Aland/Karavidopoulos/Martini/ Metzger] Novum Testamentum Graece. Begründet von Nestle, Eberhard und Nestle, Erwin. Hg. von Aland, Kurt / Aland, Barbara / Karavidopoulos, Johannes / Martini, Carlo M. / Metzger, Bruce M. 28. revidierte Auflage. Hg. vom Institut für Neutestamentliche Textforschung Münster/Westfalen und der Leitung von Strutwolf, Holger, Stuttgart 2012

Dabei verteilen sich die alttestamentlichen Bücher folgendermaßen:

[Gen = Septuaginta, Genesis ed. Wevers] Septuaginta. Vetus Testamentum

Graecum. Auctoritate Academiae Scientiarum Gottingensis editum. I. Genesis, ed. Wevers, John William, Göttingen 1974 
$[E x=$ Septuaginta, Exodus ed. Wevers $]$ Septuaginta. Vetus Testamentum Graecum. Auctoritate Academiae Scientiarum Gottingensis editum. II, 1. Exodus, ed. Wevers, John William / Quast, U(do), Göttingen 1991

$[$ Lev = Septuaginta, Leviticus ed. Quast $]$ Septuaginta. Vetus Testamentum Graecum. Auctoritate Academiae Scientiarum Gottingensis editum. II, 2. Leviticus, ed. Quast, U(do), Göttingen 1986

[Num = Septuaginta, Numeri, ed. Wevers/Quast] Septuaginta. Vetus Testamentum Graecum. Auctoritate Academiae Scientiarum Gottingensis editum. III, 1. Numeri, ed. Wevers, John William / Quast, U(do), Göttingen 1982

[Dtn = Septuaginta, Deuteronomium ed. Wevers/Quast $]$ Septuaginta. Vetus Testamentum Graecum. Auctoritate Academiae Scientiarum Gottingensis editum. III, 2. Deuteronomium, ed. Wevers, John William / Quast, $\mathrm{U}(\mathrm{do})$, Göttingen ${ }^{2} 2006$

[Ios = Septuaginta, Iosua ed. Rahlfs/Hanhart] Septuaginta, Id est Vetus Testamentum graece iuxta LXX interpretes, edidit Rahlfs, Alfred. Editio altera, quam recognovit et emendavit Hanhart, Robert. Duo volumina in uno, Stuttgart 2006, Bd. I, S. 354-405

[Idc = Septuaginta, Iudicum ed. Rahlfs/Hanhart $]$ Septuaginta, Id est Vetus Testamentum graece iuxta LXX interpretes, edidit Rahlfs, Alfred. Editio altera, quam recognovit et emendavit Hanhart, Robert. Duo volumina in uno, Stuttgart 2006, Bd. I, S. 405-495

[I. Sam = Septuaginta, I. Samuelis $(B \propto \sigma$. A) ed. Rahlfs/Hanhart] Septuaginta, Id est Vetus Testamentum graece iuxta LXX interpretes, edidit Rahlfs, Alfred. Editio altera, quam recognovit et emendavit Hanhart, Robert. Duo volumina in uno, Stuttgart 2006, Bd. I, S. 502-564

[II. Sam = Septuaginta, II. Samuelis (B $\propto \sigma$. B) ed. Rahlfs/Hanhart] Septuaginta, Id est Vetus Testamentum graece iuxta LXX interpretes, edidit Rahlfs, Alfred. Editio altera, quam recognovit et emendavit Hanhart, Robert. Duo volumina in uno, Stuttgart 2006, Bd. I, S. 565622

[I. Reg = Septuaginta, I. Regum $(B \propto \sigma$. Г) ed. Rahlfs/Hanhart] Septuaginta, Id est Vetus Testamentum graece iuxta LXX interpretes, edidit Rahlfs, Alfred. Editio altera, quam recognovit et emendavit Hanhart, Robert. Duo volumina in uno, Stuttgart 2006, Bd. I, S. 623-693

[II. Reg = Septuaginta, II. Regum $(B \propto \sigma . \Delta)$ ed. Rahlfs/Hanhart] Septuaginta, Id est Vetus Testamentum graece iuxta LXX interpretes, edidit Rahlfs, Alfred. Editio altera, quam recognovit et emendavit Hanhart, Robert. Duo volumina in uno, Stuttgart 2006, Bd. I, S. 693-752

[I. Chron = Septuaginta, I. Chronicorum ed. Rahlfs/Hanhart] Septuaginta, Id est Vetus Testamentum graece iuxta LXX interpretes, edidit Rahlfs, 
Alfred. Editio altera, quam recognovit et emendavit Hanhart, Robert. Duo volumina in uno, Stuttgart 2006, Bd. I, S. 752-811

[II. Chron = Septuaginta, II. Chronicorum ed. Hanhart] Septuaginta. Vetus Testamentum Graecum. Auctoritate Academiae Scientiarum Gottingensis editum. VII, 2. Paralipomenon liber II, ed. Hanhart, Robert, Göttingen / Bristol 2014

[Tob = Septuaginta, Tobit ed. Hanhart $]$ Septuaginta. Vetus Testamentum

Graecum. Auctoritate Academiae Scientiarum Gottingensis editum. VIII, 5. Tobit, ed. Hanhart, Robert, Göttingen 1983

[Ps = Septuaginta, Psalmi ed. Rahlfs] Septuaginta. Vetus Testamentum

Graecum. Auctoritate Academiae Scientiarum Gottingensis editum. X.

Psalmi cum Odis, ed. Rahlfs, Alfred, Göttingen ${ }^{3} 1979$, S. 81-340

[Od = Septuaginta, Odae ed. Rahlfs $]$ Septuaginta. Vetus Testamentum

Graecum. Auctoritate Academiae Scientiarum Gottingensis editum. X.

Psalmi cum Odis, ed. Rahlfs, Alfred, Göttingen ${ }^{3} 1979$, S. 341-365

[Prov = Septuaginta, Proverbia ed. Rahlfs/Hanhart $]$ Septuaginta, Id est

Vetus Testamentum graece iuxta LXX interpretes, edidit Rahlfs, Alfred.

Editio altera, quam recognovit et emendavit Hanhart, Robert. Duo volumina in uno, Stuttgart 2006, Bd. II, S. 183-238

$[\mathrm{Hi}=$ Septuaginta, Iob ed. Ziegler $]$ Septuaginta. Vetus Testamentum Graecum. Auctoritate Academiae Scientiarum Gottingensis editum. XI, 4. Iob, ed. Ziegler, Joseph, Göttingen 1982

[Cant $=$ Septuaginta, Canticum canticorum ed. Rahlfs/Hanhart $]$ Septuaginta, Id est Vetus Testamentum graece iuxta LXX interpretes, edidit Rahlfs, Alfred. Editio altera, quam recognovit et emendavit Hanhart, Robert. Duo volumina in uno, Stuttgart 2006, Bd. II, S. 260-271

[Sap = Septuaginta, Sapientia Salomonis ed. Ziegler $]$ Septuaginta. Vetus

Testamentum Graecum. Auctoritate Academiae Scientiarum Gottingensis editum. XII, 1: Sapientia Salomonis, ed. Ziegler, Joseph, Göttingen ${ }^{2} 1980$

[Sir = Septuaginta, Siracides ed. Ziegler $]$ Septuaginta. Vetus Testamentum Graecum. Auctoritate Academiae Scientiarum Gottingensis editum. XII,2. Sapientia Iesu Filii Sirach, ed. Ziegler, Joseph. 2., durchgesehene Ausgabe, Göttingen ${ }^{2} 1980$

[Hos = Septuaginta, Hosea ed. Ziegler $]$ Septuaginta. Vetus Testamentum Graecum. Auctoritate Academiae Scientiarum Gottingensis editum. XIII. Duodecim prophetae, ed. Ziegler, Joseph. 3., durchgesehene Ausgabe, Göttingen ${ }^{3} 1984$, S. 147-180

$[$ Am $=$ Septuaginta, Amos ed. Ziegler $]$ Septuaginta. Vetus Testamentum Graecum. Auctoritate Academiae Scientiarum Gottingensis editum. 
XIII. Duodecim prophetae, ed. Ziegler, Joseph. 3., durchgesehene Ausgabe, Göttingen ${ }^{3} 1984$, S. 180-205

[Mi = Septuaginta, Micha ed. Ziegler] Septuaginta. Vetus Testamentum

Graecum. Auctoritate Academiae Scientiarum Gottingensis editum. XIII. Duodecim prophetae, ed. Ziegler, Joseph. 3., durchgesehene Ausgabe, Göttingen ${ }^{3} 1984$, S. 205-227

[Ioel = Septuaginta, Joel ed. Ziegler] Septuaginta. Vetus Testamentum

Graecum. Auctoritate Academiae Scientiarum Gottingensis editum.

XIII. Duodecim prophetae, ed. Ziegler, Joseph. 3., durchgesehene Ausgabe, Göttingen ${ }^{3} 1984$, S. 228-239

$[\mathrm{Ob}=$ Septuaginta, Obadja ed. Ziegler $]$ Septuaginta. Vetus Testamentum

Graecum. Auctoritate Academiae Scientiarum Gottingensis editum. XIII. Duodecim prophetae, ed. Ziegler, Joseph. 3., durchgesehene Ausgabe, Göttingen ${ }^{3} 1984$, S. 239-243

[Nah = Septuaginta, Nahum ed. Ziegler] Septuaginta. Vetus Testamentum Graecum. Auctoritate Academiae Scientiarum Gottingensis editum. XIII. Duodecim prophetae, ed. Ziegler, Joseph. 3., durchgesehene Ausgabe, Göttingen ${ }^{3} 1984$, S. 253-260

[Hab = Septuaginta, Habakuk ed. Ziegler $]$ Septuaginta. Vetus Testamentum Graecum. Auctoritate Academiae Scientiarum Gottingensis editum. XIII. Duodecim prophetae, ed. Ziegler, Joseph. 3., durchgesehene Ausgabe, Göttingen ${ }^{3} 1984$, S. 261-275

$[$ Hag $=$ Septuaginta, Haggai ed. Ziegler $]$ Septuaginta. Vetus Testamentum Graecum. Auctoritate Academiae Scientiarum Gottingensis editum. XIII. Duodecim prophetae, ed. Ziegler, Joseph. 3., durchgesehene Ausgabe, Göttingen ${ }^{3} 1984$, S. 284-290

[Sach = Septuaginta, Sacharia ed. Ziegler] Septuaginta. Vetus Testamentum Graecum. Auctoritate Academiae Scientiarum Gottingensis editum. XIII. Duodecim prophetae, ed. Ziegler, Joseph. 3., durchgesehene Ausgabe, Göttingen ${ }^{3} 1984$, S. 291-327

[Mal = Septuaginta, Maleachi ed. Ziegler $]$ Septuaginta. Vetus Testamentum Graecum. Auctoritate Academiae Scientiarum Gottingensis editum. XIII. Duodecim prophetae, ed. Ziegler, Joseph. 3., durchgesehene Ausgabe, Göttingen ${ }^{3} 1984$, S. 328-339

[Ies = Septuaginta, Isaias ed. Ziegler] Septuaginta. Vetus Testamentum Graecum. Auctoritate Academiae Scientiarum Gottingensis editum. XIV: Isaias, ed. Ziegler, Joseph, Göttingen ${ }^{3} 1983$

[Ier $=$ Septuaginta, Jeremias ed. Ziegler $]$ Septuaginta. Vetus Testamentum Graecum. Auctoritate Academiae Scientiarum Gottingensis editum. XV. Jeremias. Baruch. Threni. Epistula Jeremiae, ed. Ziegler, Joseph, Göttingen ${ }^{3} 2006$, S. 149-450 
$[$ Bar $=$ Septuaginta, Baruch ed. Ziegler $]$ Septuaginta. Vetus Testamentum Graecum. Auctoritate Academiae Scientiarum Gottingensis editum. XV. Jeremias. Baruch. Threni. Epistula Jeremiae, ed. Ziegler, Joseph, Göttingen ${ }^{3} 2006$, S. 450-467

[Lam = Septuaginta, Lamentationes ed. Ziegler $]$ Septuaginta. Vetus Testamentum Graecum. Auctoritate Academiae Scientiarum Gottingensis editum. XV. Jeremias. Baruch. Threni. Epistula Jeremiae, ed. Ziegler, Joseph, Göttingen ${ }^{3} 2006$, S. 467-494

[Hes = Septuaginta, Ezechiel ed. Ziegler] Septuaginta. Vetus Testamentum Graecum. Auctoritate Academiae Scientiarum Gottingensis editum. XVI, 1: Ezechiel, ed. Ziegler, Joseph. Mit einem Nachtrag von Fraenkel, Detlef, Göttingen ${ }^{3} 2006$

\section{Weitere Primärliteratur bzw. Sekundärliteratur mit Edition}

[1 Clem. = Clemens Romanus, Epistula ad Corinthios (an. 95-96) ed. Jaubert (CPG 1001)] Clément de Rome, Épître aux Corinthiens. Introduction, texte, traduction, notes et index par Jaubert, Annie. Reimpression de la première édition revue et corrigée (SC 167), Paris ${ }^{2} 2000$

[Aen., dial. = Aeneas Gazaeus, Theophrastus sive de animarum immortalitate et corporum resurrectione dialogus ed. Colonna (CPG 7450)] Colonna, Maria Elisabetta (Ed.), Enea di Gaza, Teofrasto, Napoli o. J. [1958], S. 69-114

[Apc. En. = Apocalypsis Henochi ed. Black (nicht in CPG)] Black, $\mathrm{M}$ (atthew) (Ed.), Apocalypsis Henochi Graece. Fragmenta pseudepigraphorum quae supersunt Graeca. Una cum historicorum et auctorum Judaeorum Hellenistarium fragmentis, collegit et ordinavit Denis, Albert-Marie (Pseudepigrapha Veteris Testamenti Graece 3), Leiden 1970

[Apophth. Patr. $=$ Apophthegmata. Collectio aphabetica ed. Cotelier (CPG 5558)] PG 65, Sp. 71-440

[Arist., de An. = Aristoteles, de anima ed. Jannone (nicht CPG)] Aristote, De l'âme. Texte établi par Jannone, A(ntonio). Traduction et notes de Barbotin, E(dmond), Paris 1966

[Arist., EN = Aristoteles, Ethica Nicomachea ed. Bywater (nicht CPG)] Aristotelis Ethica Nicomachea, recognovit brevique adnotatione critica instruxit Bywater, I(ngram), Oxford 1979= 1894

[Bas., hex. = Basilius Caesariensis, Homiliae in hexaemeron ed. de Mendieta/Rudberg (CPG 2835)] Basilius von Caesarea, Homilien zum Hexaemeron, hg. de Mendieta, Emmanuel Amand / Rudberg, Stig Y. (GCS NF 2), Berlin 1997 
[Bas., hom. in Ps. = Basilius Caesariensis, Homiliae super psalmos ed. Garnier (CPG 2836)] PG 29, Sp. 209-228

[cat. Ac. = Catena Andreae ed. Cramer (CPG C 150)] Cramer, John Anthony, Catenae Graecorum patrum in Novum Testamentum, vol. III. Catena in Acta SS. Apostolorum, Oxford $1842=$ Hildesheim 1967

[cat. Mt. = Catena Typus $A$ in Matthaeum ed. Reuss (CPG C 110)] Matthäus-Kommentare aus der griechischen Kirche. Aus Katenenhandschriften gesammelt und hg. Reuss, Joseph (TU 61), Berlin 1957

[cat. Nicet. = Catena Typus VIII (iuxta Karo-Lietzmann) (catena Nicetae) (in Psalmos 1-150) ed. Mai (CPG C 21)] Mai, Angelo (Ed.), Novae Patrum Bibliothecae tomus tertius, continens S. Cyrilli Alexandrini commentarii in IV. Pauli epistolas et in Psalmos cum eiusdem Cyrilli et aliorum patrum fragmentis aliquot minoribus ex Vaticanis praesertim codicibus. Omnia cum latina editoris interpretatione, prologis et adnotationibus, Romae 1845, S. 139-142

[cat. Nicet. = Catena Typus VIII (iuxta Karo-Lietzmann) (catena Nicetae) (in Psalmos 1-150) ed. Mai (CPG C 21)] PG 69, Sp. 697/98-716

[Catena Lipsiensis (CPG C 2 [Édition ancienne])] Nikephoros hiero-

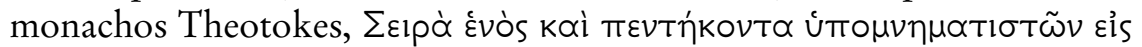

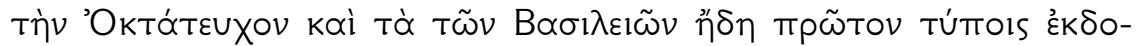

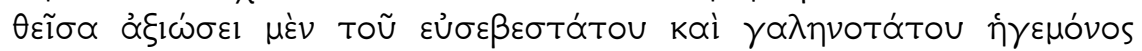

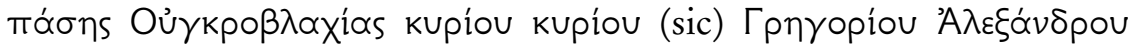

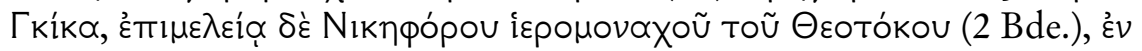

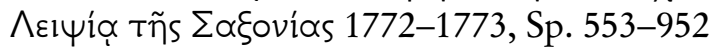

[CatGen = Catena in Genesim. Typus I et III ed. Petit (CPG C 1a und C 2a)] La Chaîne sur la Genèse. Édition intégrale. Bd. I: Chapitres 1 à 3. Bd. II: Chapitres 4 à 11. Bd. III: Chapitres 12 à 28. Bd. IV: Chapitres 29 à 50. Texte établi par Petit, Françoise (TEG 1-4), Lovanii 1991-1996

[CatEx = Catena in Exodum. Typus I et III, Fragment 1-464 ed. Petit (CPG C 1b und C 2b)] La Chaîne sur l'Exode. Édition intégrale. II. Collectio Coisliniana. III: Fonds caténique ancien (Exode 1,1 - 15,21). Texte établi par Petit, Françoise (TEG 10), Leuven / Paris / Sterling (VA) 2000, S. 45-322

[CatEx = Catena in Exodum. Typus I et III, Fragment 465-1070 ed. Petit (CPG C 1b und C 2b)] La Chaîne sur l'Exode. Édition intégrale. IV. Fonds caténique ancien (Exode 15,22 - 40,32). Texte établi par Petit, Françoise (TEG 11), Leuven / Paris / Sterling (VA) 2001

[Choricius Gazaeus, oratio VIII ed. Greco (CPG 7518)] Coricio di Gaza, Due orazioni funebri (orr. VII-VIII Foerster, Richtsteig). Introduzione, testo critico, traduzione e commento a cura di Greco, Claudia, Alessandria 2010, S. 57-80 
[Chrys., catech. series tertia $=$ Iohannes Chrysostomus, Homilia 3. Ad neophytos ed. Wenger (CPG 4467)] Jean Chrysostome, Huit catéchèses baptismales inédites. Introduction, texte critique, traduction et notes de Wenger, Antoine (SC 50bis), Paris ${ }^{2} 1970$, S. 151-167

[Chrys., hom. in Gen. = Iohannes Chrysostomus, Homiliae 1-67 in Genesim ed. Savile (CPG 4409)] PG 53, Sp. 23 - PG 54, Sp. 580

[Clem., ecl. = Clemens Alexandrinus, Eclogae propheticae ed. Nardi (CPG 1377)] Clemente Alessandrino. Estratti profettici / Eclogae propheticae, a cura di Nardi, Carlos (Biblioteca Patristica 4), Firenze 1985

[Clem., strom. = Clemens Alexandrinus, stromata ed. Stählin (CPG 1377)] Clemens Alexandrinus, Werke. Bd. 3: Stromata, Buch VII und VIII. Excerpta ex Theodoto - Eclogae propheticae - Quis dives salvetur Fragmente, ed. Stählin, Otto. In 2. Auflage neu hrsg. Früchtel, Ludwig, Berlin 1970

[CollCoisl $\mathrm{Ex}=$ Collectio exegetica Coisliniana in Octateuchum et Regnorum libros. In Exodum ed. Petit (CPG C 5)] La Chaîne sur l'Exode. Édition intégrale. II. Collectio Coisliniana. III: Fonds caténique ancien (Exode 1,1 - 15,21). Texte établi par Petit, Françoise (TEG 10), Lovanii 2000, S. 1-44

[Cosm. Ind., top. = Cosmas Indicopleustes, Topographia christiana ed. Wolska-Conus (CPG 7468)] Cosmas Indicopleustès, Topographie Chrétienne. Introduction, texte critique, illustration, traduction et notes Wolska-Conus, Wanda (3 Bde.) (SC 141, 159, 197), Paris 1968-1973

[Cyr. H., catech. 1-18 = Catecheses ad illuminandos 1-18 ed. Reischl (CPG 3585 [2])] Reischl, Guilelmus Carolus [i. e. Wilhelm Carl] (Ed.), St. Cyrilli Hierosolymorum archiepiscopi opera quae supersunt omnia. Bd. 1. Ad libros mss. et impressos recensuit, notis criticis commentariis indicibusque locupletissimis illustravit, Monaci 1848

[Cyr., ador. = Cyrillus Alexandrinus, De adoratione et cultu in spiritu et ueritate ed. Aubert (CPG 5200)] PG 68, Sp. 133-1125

[Cyr., Am. $=$ Cyrillus Alexandrinus, Commentarius in xii prophetas minores (Amos) ed. Pusey (CPG 5204)] Sancti patris nostri Cyrilli archiepiscopi Alexandrini In XII prophetas. Post Pontanum et Aubertum edidit Pusey, Philippus Eduardus. Vol. I, Oxonii 1848, S. 366-545

[Cyr., comm. Lc. = Cyrillus Alexandrinus, Commentarii in Lucam. (3) Scholia in catenis ed. Mai (CPG 5207 [3])] PG 72, Sp. 476-949

[Cyr., glaph. Ex. = Cyrillus Alexandrinus, Glaphyra in Pentateuchum ed. Aubert (CPG 5201)] PG 69, Sp. 385-537

[D. S. = Diodorus Siculus, Bibliotheca historica ed. Casevitz (nicht in CPG)] Diodore de Sicile, Bibliothèque historique. Tome V. Livre V. Livre de 
îles. Texte établi et traduit Casevitz, Michel. Présenté et commenté Jaquemin, Anne, Paris 2015

[Didym., 2 Cor. = Didymus Alexandrinus, Fragmenta in Epistulam ii ad Corinthios ed. Staab (CPG 2560)] Staab, Karl (Ed.), Pauluskommentare aus der griechischen Kirche. Aus Katenenhandschriften gesammelt und hg. (NTA 15), Münster 1933, S. 14-44

[Didym., EcclT 1,1 - 2,14 = Didymus Alexandrinus, Commentarii in Ecclesiasten in chartis papyraceis Turanis 1,1 - 2,14 ed. Binder/Liesenborghs (CPG 2555 [1])] Didymus der Blinde, Kommentar zum Ecclesiastes (Tura-Papyrus). Teil I: Kommentar zu Eccl. Kap. 1,1 - 2,14 (Einleitung, Text, Übersetzung, Indices), in Zusammenarbeit mit dem Ägyptischen Museum zu Kairo hg. und übers. von Binder, Gerhard / Liesenborghs, Leo (PTA 25), Bonn 1979

[Didym., EcclT 3 - 4,12 = Didymus Alexandrinus, Commentarii in Ecclesiasten 3-4,12 in chartis papyraceis Turanis ed. Gronewald (CPG 2555 [2])] Didymus der Blinde, Kommentar zum Ecclesiastes (TuraPapyrus). Teil II. Kap. 3 - 4,12, in Zusammenarbeit mit dem Ägyptischen Museum zu Kairo hg. und übers. von Gronewald, Michael (PTA 22), Bonn 1977

[Didym., EcclT 11-12 = Didymus Alexandrinus, Commentarii in Ecclesiasten in chartis papyraceis Turanis. In Ecclesiasten 11 - 12 ed. Binder/Liesenborghs (CPG 2555 [6])] Didymus der Blinde, Kommentar zum Ecclesiastes (Tura-Papyrus). Teil VI: Kommentar zu Eccl. Kap. 11-12. In Zusammenarbeit mit dem Ägyptischen Museum zu Kairo unter der Mitwirkung von Koenen, Ludwig hg. und übers. von Binder, Gerhard / Liesenborghs, Leo (PTA 9), Bonn 1969

[Didym., Ex. = Didymus Alexandrinus, Commentarii in Octateuchum et Reges ed. Devreesse (CPG 2546 [2])] Devreesse, Robert, Les anciens commentateurs Grecs de l'Octateuque et des Rois (Fragments tirés des chaînes) (StT 201), Città del Vaticano 1959, S. 172

[Didym., Ex. = Didymus Alexandrinus, Commentarii in Octateuchum et Reges ed. Nikephoros Theotokes (CPG 2546 [1])] PG 39, Sp. 1113-1116 [Didym., Gen. = Didymus Alexandrinus, Commentarii in Octateuchum et Reges ed. Nautin/Doutreleau (CPG 2546 [1])] Didyme l'aveugle, Sur la Genèse. Texte inédit d'après un papyrus de Toura. Introduction, édition, traduction et notes par Nautin, Pierre, avec la collaboration de Doutreleau, Louis (SC 233/244), Paris 1976-1978

[Didym., Hi. = Didymus Alexandrinus, Commentarii in Iob in chartis papyr. Turanis ed. Hagedorn/Hagedorn (CPG 2553 [4])] Hagedorn, Dieter / Hagedorn, Ursula (Edd.), Die älteren griechischen Katenen zum Buch Hiob (PTS 40.48.53.59), Berlin / New York 1994-2004 
[Didym., Hi. = Didymus Alexandrinus, Commentarii in Iob ed. Young (nicht in CPG)] PG 39, Sp. 1120-1154

[Didym., HiT 1-4 = Didymus Alexandrinus, Commentarii in Iob in chartis papyraceis Turanis ed. Henrichs (CPG 2553 [1])] Didymus der Blinde, Kommentar zu Hiob. Teil I. Kommentar zu Hiob Kap. 1-4, in Zusammenarbeit mit dem Museum zu Kairo hg. und übers. von Henrichs, Albert (PTA 1), Bonn 1968

[Didym., HiT 5,1 - 6,29 = Didymus Alexandrinus, Commentarii in Iob in chartis papyraceis Turanis ed. Henrichs (CPG 2553 [2])] Didymus der Blinde, Kommentar zu Hiob. Teil II. Kommentar zu Hiob Kap. 5,1 6,29, in Zusammenarbeit mit dem Museum zu Kairo hg., übers. und erläutert von Henrichs, Albert (PTA 2), Bonn 1968

[Didym., HiT 7,20c - 11 = Didymus Alexandrinus, Commentarii in Iob in chartis papyraceis Turanis ed. Hagedorn/Hagedorn/Koenen (CPG 2553 [3])] Didymus der Blinde, Kommentar zu Hiob. Teil III. Kommentar zu Hiob Kap. 7,20c - 11, in Zusammenarbeit mit dem Ägyptischen Museum zu Kairo hg., übers., erläutert von Hagedorn, Ursula / Hagedorn, Dieter / Koenen, Ludwig (PTA 3), Bonn 1968

[Didym., HiT 12,1 - 16,8a = Didymus Alexandrinus, Commentarii in Job in chartis papyraceis Turanis ed. Hagedorn/Hagedorn/Koenen (CPG 2553 [4])] Didymus der Blinde, Kommentar zu Hiob (Tura-Papyrus). Teil IV.1. In Zusammenarbeit mit dem Ägyptischen Museum zu Kairo hg. und übers. von Hagedorn, Dieter / Hagedorn, Ursula / Koenen, Ludwig (PTA 33/1), Bonn 1985

[Didym., Ps. = Didymus Alexandrinus, Fragmenta in psalmos (e commentario altero) ed. Mühlenberg (CPG 2551)] Mühlenberg, Ekkehard, Psalmenkommentare aus der Katenenüberlieferung (2 Bde.) (PTS 1516), Berlin / New York 1975-1977

[Didym., PsT 20-21 = Commentarii in psalmos in chartis papyraceis Turanis in ps. Ps. 20 - 21 ed. Doutreleau/Gesché/Gronewald (CPG 2550 [1])] Didymus der Blinde, Psalmenkommentar (Tura-Papyrus). Teil I: Kommentar zu Psalm 20 - 21, hg. und übers. von Doutreleau, Louis / Gesché, Adolphe / Gronewald, Michael (PTA 7), Bonn 1969

[Didym., PsT 22 - 26,10 = Didymus Alexandrinus, Commentarii in psalmos in chartis papyraceis Turanis in ps. Ps. $22-26,10$ ed. Gronewald (CPG 2550 [2])] Didymus der Blinde, Psalmenkommentar (Tura-Papyrus). Teil II: Kommentar zu Psalm 22 - 26,10, hg. und übers. von Gronewald, Michael (PTA 4), Bonn 1968

[Didym., PsT 29-34 = Didymus Alexandrinus, Commentarii in psalmos in chartis papyraceis Turanis in ps. 29 - 34 ed. Doutreleau/Gesché/Gronewald (CPG 2550 [3])] Didymus der Blinde, Psalmenkommentar (Tura- 
Papyrus). Teil III: Kommentar zu Psalm 29 - 34, in Verbindung mit Gesché, A(dolphe), hg. und übers. von Gronewald, Michael (PTA 8), Bonn 1969

[Didym., PsT 35-39 = Didymus Alexandrinus, Commentarii in psalmos in chartis papyraceis Turanis in ps. $35-39$ ed. Gronewald (CPG 2550 [4])] Didymus der Blinde, Psalmenkommentar. Teil IV: Kommentar zu Psalm 35-39, hg. und übers. von Gronewald, Michael (PTA 6), Bonn 1969

[Didym., ZachT $=$ Didymus Alexandrinus, Commentarium in Zacchariam ed. Doutreleau (CPG 2549)] Didyme l'Aveugle, Sur Zacharie. Texte inédit d'apres un papyrus de Toura. Introduction, texte critique, traduction et notes Doutreleau, Louis (SC 83-85), Paris 1962

[Diod., Ex. = Diodorus Tarsensis, Fragmenta in Ocateuchum ed. Deconinck (CPG 3815)] Deconinck, Joseph, Essai sur la chaîne de l'Octateuque avec une édition des Commentaires de Diodore de Tarse, Paris 1912, S. $134 *-148 *$

[Diod., fr. in Ex. = Diodorus Tarsensis, fragmenta in Exodum (latine) ed. Pitra (CPG 3817)] Spicilegium Solesmense, complectens sanctorum patrum scriptorumque ecclesiasticorum anecdota hactenus opera, selecta e Graecis orientalibusque et latinis codicibus ed. Pitra, J(ean-)B(aptiste). Bd. 1. In quo praecipue auctores saeculo $\mathrm{V}$ antiquiores proferuntur et illustrantur, Parisiis 1852, S. 269-275

[Epiph., haer. 1-33 = Epiphanius Constantiensis, Panarion (Adversus haereses) 1-33 ed. Holl/Bergermann/Collatz (CPG 3745)] Epiphanius, I. Ancoratus und Panarion, hg. Holl, Karl. Zweite, erweiterte Auflage hg. Bergermann, Marc / Collatz, Christian Friedrich. Teilband I/1: Text. Mit einem Geleitwort von Markschies, Christoph. Teilband I/2: Addenda et Corrigenda. Mit einem Anhang zu den Randbemerkungen Melanchthons im Jenensis Ms. Bos. f. 1 von Dummer Jürgen / Markschies, Christoph (GCS NF 10/1 bzw. 2 Epiphanius 1), Berlin 2013

[Epiph., mens. = Epiphanius Constantiensis, De mensuris et ponderibus ed.

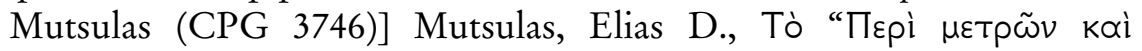

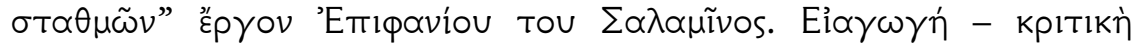

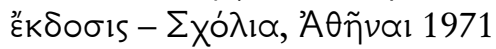

[Epiph., mens. = Epiphanius Constantiensis, De mensuris et ponderibus ed. Migne (CPG 3746)] PG 43, Sp. 237-293

[Epiph., mens. (exc. Graec. 4) = Epiphanius Constantiensis, De mensuris et ponderibus (excerptum Graecum 4) ed. Hultsch (CPG 3746)] Metrologicorum scriptorum reliquiae, vol. I, quo scriptores Graeci continentur, collegit, recensuit, partim nunc primum ed. Hultsch, Fridericus, Lipsiae 1864, S. 259-267 
[Et. Gud. = Etymologicum Gudianum ed. Sturz (nicht in CPG)] Etymologicum Graecae Linguae Gudianvm et alia grammaticorum scripta e codicibus manuscriptis nunc primum edita. Accedunt notae ad Etymologicon magnum ineditae E. H. Barkeri, Imm. Bekkeri [...] aliorumque. Cum indice locupletissimo, quas digessit et una cum suis ed. Sturzius, Frider. Gul., Hildesheim 1973 = Lipsiae 1818

[Eus., ecl. = Eusebius Caesariensis, Generalis elementaria introductio ('H

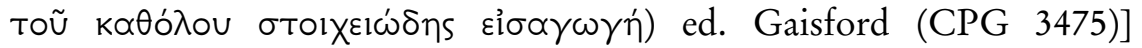
Eusebii Pamphili episcopi Caesariensis Eclogae propheticae e codice manuscripto Bibliothecae caesareae Vindobonensis nunc primum edidit Gaisford, Thomas, Oxonii 1847

[Eus., fr. = Eusebius Caesariensis, Generalis elementaria introductio ('H тоũ

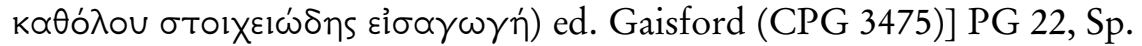
1017-1262

[Eus., p. e. = Eusebius Caesariensis, Praeparatio evangelica ed. Mras/des Places (CPG 3486)] Eusebius, Werke. 8. Bd.: Die Praeparatio evangelica, ed. Mras, Karl. 2., bearbeitete Auflage, hg. des Places, Eduard (GCS 43 Eusebius 8/1), Berlin 1982

[Eus. Em. = Eusebius Emesenus, Commentarii in Octatenchum et Reges ed. Buytaert (CPG 3532)] Buytaert, É(lio) M(arie), L’héritage littéraire d'Eusèbe d'Émèse. Étude critique et historique. Textes (BMus 24), Louvain 1949

[Eus. Em. = Eusebius Emesenus, Commentarii in Octatenchum et Reges ed. Devreesse (CPG 3532)] Devreesse, Robert, Les anciens commentateurs Grecs de l'Octateuque et des Rois (Fragments tirés des chaînes) (StT 201), Città del Vaticano 1959, S. 55-103

[Eus. Em. (armen.) = Eusebius Emesenus, Commentarii in Octateuchum (armen.) ed. Yovhannêsean (CPG 3542)] Ewsebios Emesac'i, Meknowt'iwnk' owt'amatean groc' astowacašnzin. Ašxatasirowt'eamb Yovhannêsean, Vahan = Commentaire de l'octateuque (Bibliothèque de l'Académie Arménienne de Saint Lazare), Venetik 1980

[Eus. Em., de Mos. = Eusebius Emesenus, Sermones $i$-xii (Collectio Trecensis). De Moyse ed. Buytaert (CPG 3525 [12])] Buytaert, Élio Marie, Eusèbe d'Émèse, Discours conservés en latin. Textes en partie inédits. Bd. 1: La collection de Troyes (Spicilegium sacrum Lovaniense. Études et documents 26), Louvain 1953, S. 277-289: [XII] De Moyse

[Eust., Laz. = Ps.-Eustathius Antiochenus, Homilia christologica in Lazarum, Mariam et Martham ed. Cavallera (CPG 3394)] Cavallera, Ferdinandus (Ed.), S. Eustathii Episcopi Antiocheni in Lazarum, Mariam et Martham homilia christologica. Nunc primum e cod. 
gronouiano edita cum commentario de fragmentis eustathianis, acesserunt fragmenta Flauiani I Antiocheni, Parisiis 1905

[Field = Origenes, Hexapla ed. Field (CPG 1500)] Origenis Hexaplorum quae supersunt; sive veterum interpretum Graecorum in totum Vetus testamentum fragmenta, [...] concinnavit, emendavit, et multis partibus auxit Field, Fridericus, Oxonii 1875

[Gal., simpl. med. temp. ac. fac. = Gal., De simplicium medicamentorum temperamentis ac facultatibus libri xi ed. Kühn (nicht CPG)] Medicorum Graecorum opera quae exstant, editionem curavit Kühn, C(arl) G(ottlob). Vol. 11 Claudii Galeni opera omnia tom. 11, Hildesheim 1965 = Leipzig 1826, S. 379-892

[Gr. Naz., or. $28=$ Gregorius Nazianzenus, Orationes $x l v$. Theologica $I I^{a}$ vel De theologia ed. Gallay/Jourjon (CPG 3010 [28])] Grégoire de Nazianze, Discours 27-31 (Discours théologiques). Introduction, texte critique, traduction et notes par Gallay, Paul, avec la collaboration de Jourjon, Maurice, Paris 1978, S. 100-174

[Gr. Naz., or. 28 = Gregorius Nazianzenus, Oratio 28 (Theologica II ${ }^{a}$ vel De theologia) ed. Caillau-Clémencet (CPG 3010 [28])] PG 36, Sp. 25-72

[Gr. Naz., or. $45=$ Gregorius Nazianzenus, Orationes xlv ed. CaillauClémencet (CPG 3010 [45])] PG 35, Sp. 396 - PG 36, Sp. 664, hier PG 36, Sp. 624-664

[Gr. Nyss., hom. opif. = Gregorius Nyssenus, De opificio hominis ed. Forbes (CPG 3154)] Sancti patris nostri Gregorii Nysseni, Basilii magni fratris, quae supersunt omnia [...] ed. Georgius H. Forbesius. Tomus I, Burntisland 1855, S. 102-318

[Gr. Nyss., hom. opif. = Gregorius Nyssenus, De opificio hominis ed. Migne (CPG 3154)] PG 44, Sp. 124-256

[Gr. Nyss., v. Mos. = Gregorius Nyssenus, De vita Moysis ed. Simonetti (CPG 3159)] Simonetti, Manlio (Ed.), Gregorio di Nissa, La Vita di Mosè (Scrittori greci e latini), Milano ${ }^{4} 2011$

[Gr. Nyss., v. Mos. = Gregorius Nyssenus, De vita Moysis ed. Daniélou (CPG 3159)] Grégoire de Nysse, La vie de Moïse ou Traité de la perfection en matière de vertu. Introduction, texte critique et traduction de Daniélou, Jean, 3. édition, revue et corrigée (SC 1ter), Paris 1968

[Gr. Nyss., v. Mos. = Gregorius Nyssenus, De vita Moysis ed. Migne (CPG 3159)] PG 44, Sp. 297-430

[Hieron., epp. = Hieronymus presbyter, Epistulae ed. Hilberg (CPL 620)] Sancti Hieronymi Epistulae, rec. Hilberg, Isidorus. Pars I: Epistulae ILXX (Corpus scriptorum ecclesiasticorum Latinorum 54), Vindobonae / Lipsiae 1910 
[Hom., Il. = Homerus, Ilias ed. West (nicht in CPG) $]$ Homeri Ilias, recensuit / testimonia congessit West, M. L. (BiTeu), Stutgardiae / Lipsiae 1998-2000

[Hom., Od. = Homerus, Odyssea ed. van Thiel (nicht in CPG)] Homeri Odyssea, recognovit van Thiel, Helmut (Bibliotheca Weidmanniana), Hildesheim / Zürich / New York 1991

[Iren., fr. 24 = Irenaeus Lugdonensis, Fragmenta uaria graeca ed. Harvey (CPG 1315 [12])] Harvey, W(illiam) Wigan, Sancti Irenaei episcopi Lugdunensis Libros quinque adversus Haereses [...] Bd. II, Cantabrigiae 1857, S. 491

[Išo'dad von Merv = Išo'dad von Merv, Kommentar zum Alten Testament ed. Van den Eynde (nicht in CPG)] Commentaire d'Išo'dad de Merv sur l'Ancien Testament. Exode - Deutéronome, éd. Van den Eynde, Ceslas (CSCO 176 syr. 80), Louvain 1958

[Išo'dad von Merv = Išo'dad von Merv, Kommentar zum Alten Testament übers. Van den Eynde (nicht in CPG)] Commentaire d'Išo'dad de Merv sur l'Ancien Testament. Exode - Deutéronome, trad. Van den Eynde, C(eslas) (CSCO 179 syr. 81), Louvain 1958

[Iust., dial. = Dialogus cum Tryphone Indaeo ed. Marcovich (CPG 1076)] Iustini Martyris Dialogus cum Tryphone, ed. Marcovich, Miroslav (PTS 47), Berlin 1997

[J., AJ = Flavius Josephus, Antiquitates Indaicae ed. Niese (nicht in CPG)] Flavii Josephi Opera I-IV edidit et apparatu critico instruxit Niese, Benedikt, Berolini $1955=1887$

[J., AJ I-III = Flavius Josephus, Antiquitates Indaicae I-III ed. Nodet (nicht in CPG)] Flavius Josèphe, Les antiquités juives. Texte, traduction et notes par Nodet, Etienne avec la collaboration de Berceville, Gilles / Waschawski, Elisabeth, Paris ${ }^{3} 2000$

[J., Ap. I = Flavius Josephus, Contra Apionem liber I ed. Labow (nicht in CPG)] Flavius Josephus, Contra Apionem. Buch I: Einleitung, Text, textkritischer Apparat, Übersetzung und Kommentar Labow, Dagmar (Beiträge zur Wissenschaft vom Alten und Neuen Testament 167), Stuttgart 2005

[Jo. D., parall. = Iohannes Damascenus, Sacra parallela. Recensiones secundum alphabeti litteras dispositae, quae tres libros conflant ed. Lequien (CPG 8056 [2])] PG 95, Sp. 1041-1588

[Jub. = Jubiläenbuch, übers. Vanderkam (nicht in CPG)] The Book of Jubilees, translated Vanderkam, James C. (CSCO.Ae 511/88), Lovanii 1989

[Komm. v. Diyarbakır (syr.) = Kommentar von Diyarbakır (syr.) ed. Van Rompay (nicht in CPG)] Van Rompay, Lucas (Ed., Übers.), Le 
commentaire sur Genèse-Exode 9,32 du manuscrit (olim) Diyarbakır 22 (CSCO.S 483-484/205-206), Lovanii 1986

Leseausgabe Origenes [entspricht Or., comm. in Gen. übers. Metzler und Or., schol. in Gen. übers. Metzler] = Origenes, Werke mit deutscher Übersetzung, hg. Fürst, Alfons / Markschies, Christoph. Band 1/1. Die Kommentierung des Buches Genesis. Eingeleitet, übersetzt und erläutert von Metzler, Karin, Berlin / New York 2010 [eine kritische Edition wird in der Reihe GCS erscheinen; der Textbestand insgesamt wird nicht mit dem der Leseausgabe identisch sein, aber die hier genannten Fragmentnummern werden sich nicht ändern]

[Midrash shemot Rabbah ed. Shinan (nicht in CPG)] Midrash shemot Rabbah. A Critical Edition Based on a Jerusalem Manuscript with Variants, Commentary and Introduction Shinan, Avigdor, ‘Jerusalem / Tel Aviv 1984

[Nicaeno-Constantinopolitanum $=$ Concilium oecumenicum Constantinopolitanum I. Symbolum ed. Dossetti (CPG 8599)] Dossetti, Giuseppe Luigi (Ed.), Il simbolo di Nicea e di Costantinopoli. Edizione critica. Ricerca condotta col contributo del Consiglio Nazionale delle Ricerche (TRSR 2), Roma / Freiburg / Basel / Barcelona / Wien 1967, S. 244-251

[Nicaenum $=$ Concilium Oecumenicum Nicaenum. Symbolum ed. Dossetti (CPG 8512)] Dossetti, Giuseppe Luigi (Ed.), Il simbolo di Nicea e di Costantinopoli. Edizione critica. Ricerca condotta col contributo del Consiglio Nazionale delle Ricerche (TRSR 2), Roma / Freiburg / Basel / Barcelona / Wien 1967, S. 226-241

[Or. (dub.), Ps. = Origenes, Libri (tomi) in Psalmos ed. Pitra (CPG 1426 [II 5])] Pitra, Joannes Baptista (Ed.), Analecta sacra Spicilegio Solesmensi parata. Bd. III: Patres antenicaeni, Farnborough 1966 = Veneto 1883, Bd. II: S. 444-483, III: S. 1-364

[Or., adnot. in Ex. = Origenes, In Exodum excerpta (fragmenta). In catenis (CPG 1413 [2])] PG 17, Sp. 16f.

[Or., Cant. $=$ Origenes, Libri $x$ in Canticum canticorum (latine, interprete Rufino) ed. Baehrens (CPG 1433)] Origenes, Werke. Bd. 8. Homilien zu Samuel I, zum Hohelied und zu den Propheten. Kommentar zum Hohelied in Rufins und Hieronymus' Übersetzungen, ed. Baehrens, W(ilhelm) A(dolf) (GCS 33 Origenes 8), Leipzig 1925

[Or., Cant. (fr. in catenis) = Origenes, Libri $x$ in Canticum canticorum. Fragmenta graeca ed. Barbàra (CPG 1433)] Barbàra, Maria Antonietta (Ed.), Commento di Origene al Cantico dei cantici. Testi in Lingua Greca. Introduzione, testo, traduzione e commento (Biblioteca patristica), Bologna 2005 
[Or., Cels. = Origenes, Contra Celsum ed. Borret (CPG 1476)] Origène, Contre Celse. Introduction, texte critique, traduction et notes par Borret, Marcel (SC 132.136.147.150), Paris 1961-1971

[Or., comm. in Gen. = Origenes, Commentarii in Genesim (fragmenta) übers. Metzler (CPG 1410 [1-6]); zitiert als „Leseausgabe Origenes“] Origenes, Werke mit deutscher Übersetzung, hg. Fürst, Alfons / Markschies, Christoph. Band 1/1. Die Kommentierung des Buches Genesis. Eingeleitet, übersetzt und erläutert von Metzler, Karin, Berlin / New York 2010

[Or., comm. in I Cor. = Fragmenta e catenis in Epistulam primam ad Corinthios ed. Jenkins (CPG 1458)] Jenkins, Claude (Ed.), Origen on I Corinthians, in: JThS 9 (1907/1908), S. 231-247; 353-372; 500-514; 10 (1909), S. 29-51

[Or., comm. in Mt. XI = Teil von Origenes, Commentarii in Matthaeum. Libri X-XVII (in Mt. 13,36-22,33) (graece) ed. Girod (CPG 1450 [1])] Origène, Commentaire sur l'évangile selon Matthieu. Bd. 1: Livres XXI. Introduction, traduction et notes par Girod, Robert (SC 162), Paris 1970

[Or., comm. ser. 1-145 in Mt. = Origenes, Commentarii in Matthaeum. Commentariorum series (latine) (in Mt. 22,34 - 27,63) ed. Klostermann/Benz/Treu (CPG 1450 [2])]: Origenes, Werke. 11. Bd.: Matthäuserklärung. II. Die lateinische Übersetzung der Commentariorum series. 2. bearbeitete Auflage hg. von Treu, Ursula (GCS 38 Origenes 11,2) = ${ }^{2} 1976$

[Or., fr. 1-112 in Lc. = Commentarii in Lucam (fragmenta) ed. Rauer (CPG 1452)] Origenes, Werke. Bd. 9: Die Homilien zu Lukas in der Übersetzung des Hieronymus und die griechischen Reste der Homilien und des Lukas-Kommentars, ed. Rauer, Max (GCS 49 Origenes 9), Berlin ${ }^{2} 1959$, S. 227-336

Or., hom. 1-9 in Is. = Origenes, In Isaiam homiliae xxxii (latine, interprete Hieronymo) ed. Baehrens (CPG 1437)] Origenes, Werke. Bd. 8. Homilien zu Samuel I, zum Hohelied und $\mathrm{zu}$ den Propheten. Kommentar zum Hohelied in Rufins und Hieronymus' Übersetzungen, ed. Baehrens, W(ilhelm) A(dolf) (GCS 33 Origenes 8), Leipzig 1925, S. $242-289$

[Or., hom. 1-13 in Ex., lat. = Origenes, In Exodum bomiliae XIII (lat.) ed. Baehrens (CPG 1414)] Origenes, Werke. 6. Bd.: Homilien zum Hexateuch in Rufins Übersetzung, ed. Baehrens, W(ilhelm) A(dolf) (GCS 29 Origenes 6), Leipzig 1920, S. 145-279

[Or., hom. 1-16 in Gen., lat. = Origenes, In Genesim homiliae xvi (latine, Rufino interprete) ed. Habermehl (CPG 1411)] Origenes, Werke. 6. Bd.: 
Homilien zum Hexateuch in Rufins Übersetzung. Teil 1: Die Homilien zu Genesis (Homiliae in Genesis), ed. Habermehl, Peter (GCS NF 17). Zweite, völlig überarbeitete Auflage, Berlin / Boston 2012

[Or., hom. 1-16 in Lev. = Origenes, In Leviticum homiliae xvi (latine, interprete Rufino) ed. Baehrens (CPG 1416)] Origenes, Werke. 6. Bd.: Homilien zum Hexateuch in Rufins Übersetzung. Erster Teil: Die Homilien zu Genesis, Exodus und Leviticus, ed. Baehrens, W(ilhelm) A(dolf) (GCS 29 Origenes 6), Leipzig 1920, S. 280-507

[Or., hom. 1-20 in Jer. = Origenes, Homiliae in Ieremiam. Homiliae $x x$ graecae ed. Klostermann/Nautin (CPG 1438 [1])] Origenes, Werke. 3. Bd.: Jeremiahomilien. Klageliederkommentar. Erklärung der Samuelund Königsbücher hg. Klostermann, Erich. 2., bearbeitete Auflage, hg. Nautin, Pierre (GCS 6 Origenes 3), Berlin 1983, S. 1-232

[Or., hom. 1-39 in Lc. = Origenes, In Lucam homiliae xxix (latine, Hieronymo interprete) ed. Rauer (CPG 1452)] Origenes, Werke. 9. Bd.: Die Homilien zu Lukas in der Übersetzung des Hieronymus und die griechischen Reste der Homilien und des Lukas-Kommentars, ed. Rauer, Max (GCS 49 Origenes 9), Berlin 21959, S. 1-222

[Or., hom. in Ps. = Origenes, Homiliae de Psalmis ed. Perrone/Cacciari/ Prinzivalli (CPG 1428)] Origenes, Werke. 13. Bd. Die neuen Psalmenhomilien. Eine kritische Edition des Codex Monacensis Graecus 314, hg. von Perrone, Lorenzo in Zusammenarbeit mit Molin Pradel, Marina / Prinzivalli, Emanuela / Cacciari, Antonio (GCS NF 19), Berlin / München / Boston 2015

[Or. in: Gr. Naz. / Bas. (?), philoc. = Gregorius Nazianzenus / Basilius Caesareensis (?), philocalia 21-27 ed. Junod (CPG 1410 [3a])] Origène, Philocalie 21-27. Sur le libre arbitre. Introduction, texte, traduction et notes par Junod, Éric (SC 226), Paris 1976

[Or. in: Gr. Naz. / Bas. (?), philoc. = Gregorius Nazianzenus / Basilius Caesareensis (?), philocalia ed. Robinson (CPG 1502)] The Philocalia of Origen. The text revised with a critical introduction and indices by Robinson, J. Armitage, Cambridge 1893

[Or., Jo. I, II und XXXII = Origenes, Commentarii in Ioannem ed. Preuschen (CPG 1453) ed. Preuschen] Origenes, Werke. Vierter Bd.: Der Johanneskommentar, hg. Preuschen, Erwin (GCS 10 Origenes 4), Leipzig 1903

[Or., Jo. X = Origenes, Commentarii in Ioannem X ed. Blanc (CPG 1453)] Origène, Commentaire sur Saint Jean. Tome II: Livres VI et X. Texte grec, avant-propos, traduction et notes par Blanc, Cecile (SC 157), Paris 1970 
[Or., mart. = Origenes, Exhortatio ad Martyrium ed. Koetschau (CPG 1475)] Origenes, Werke. Erster Bd.: Die Schrift vom Martyrium. Buch I-IV gegen Celsus, hg. Koetschau, Paul (GCS 3 Origenes 1), Leipzig 1899, S. 3-42

[Or., or. = Origenes, de oratione ed. Koetschau (CPG 1477)] Origenes, Werke. Zweiter Bd.: Buch V-VII gegen Celsus. Die Schrift vom Gebet, hg. Koetschau, Paul (GCS 3 Origenes 2), Leipzig 1899

[Or., pasch. = Origenes, De Pascha ed. Guéraud/Nautin (CPG 1480)] Origène, Sur la Pâque. Traité inédit publié d'après un papyrus de Toura par Guéraud, Octave / Nautin, Pierre (CAnt 2), Paris 1979

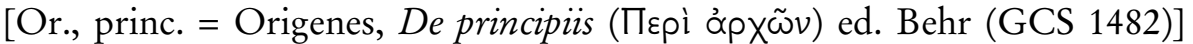
Origen, On First Principle. Edited and translated by Behr, John (Oxford Early Christian Texts) (2 Bde.), Oxford 2017

[Or., Ps. = Origenes, Libri (tomi) in Psalmos ed. Harl (CPG 1462 [6] und C 24)] La chaine Palestinienne sur le psaume 118 (Origène, Eusèbe, Didyme, Apollinaire, Athanase, Théodoret) (SC 189.190), Paris 1972

[Or., schol. in Gen. = Origenes, Scholia in Genesim übers. Metzler (CPG 1410 [5] und 1412); zitiert als „Leseausgabe Origenes“] Origenes, Werke mit deutscher Übersetzung, hg. Fürst, Alfons / Markschies, Christoph. Band 1/1. Die Kommentierung des Buches Genesis. Eingeleitet, übersetzt und erläutert von Metzler, Karin, Berlin / New York 2010

[Or., sel. in Ex. = Origenes, In Exodum excerpta (fragmenta), angezeigt von Devreesse (CPG 1413 [2])] Devreesse, Robert, Les anciens commentateurs Grecs de l'Octateuque et des Rois (Fragments tirés des chaînes) (StT 201), Città del Vaticano 1959, S. 41-44

[Or., sel. in Ex. = Origenes, In Exodum excerpta (fragmenta) (CPG 1413 [2])] PG 12, Sp. 281-297

[Or., sel. in Ezech. = Origenes, Fragmenta in catenis (incertum e quo opere) ed. Delarue (CPG 1442)] PG 13, Sp. 768-825 und 288

[Ph., Abrah. = Philo Judaeus, De Abrabamo ed. Cohn (nicht in CPG)] Philonis Alexandrini Opera quae supersunt vol. IV ed. Cohn, Leopoldus, Berolini $1962=1902$, S. 1-60

[Ph., agric. = Philo Judaeus, de agricultura ed. Wendland (nicht in CPG)] Philonis Alexandrini Opera quae supersunt vol. II ed. Wendland, Paulus, Berolini 1962 = 1897, S. 95-132

[Ph., Decal. = Philo Judaeus, de decalogo ed. Cohn (nicht in CPG)] Philonis Alexandrini Opera quae supersunt vol. IV ed. Cohn, Leopoldus / Wendland, Paulus, Berolini 1962 = 1902, S. 269-307

[Ph., fragmenta $=$ Philo Judaeus, fragmenta ed. Harris (nicht in CPG)] Fragments of Philo Iudaeus, newly edited by Harris, James Rendel. With two facsimiles, Cambridge 1886 
[Ph., migr. = Philo Judaeus, de migratione Abrabam ed. Cadiou (nicht in CPG)] Philon d'Alexandrie, La migration d'Abraham. Introduction, texte critique, traduction et notes Cadiou, René (SC 47), Paris 1957

[Ph., post. Caini = Philo Judaeus, De posteritate Caini ed. Wendland (nicht in CPG)] Philonis Alexandrini Opera quae supersunt vol. II ed. Wendland, Paulus, Berolini $1962=1897$, S. 1-41

[Ph., quaest. Ex. = Philo Judaeus, Quaestiones in Exodum ed. Petit (nicht in CPG)] Philon d'Alexandrie, Cuvres. Bd. 33: Quaestiones in Genesim et in Exodum. Fragmenta Graeca. Introduction, texte critique et notes par Petit, Françoise, Paris 1978, S. 228-306

[Ph., quaest. Ex. = Philo Judaeus, Quaestiones in Exodum ed. Wendland (nicht in CPG)] Wendland, Paul, Neu entdeckte Fragmente Philos. Nebst einer Untersuchung über die ursprüngliche Gestalt der Schrift De sacrificiis Abelis et Caini, Berlin 1891

[Ph., quaest. Ex. (armen.) = Philo Judaeus, Quaestiones in Exodum (armen.) übers. Mercier/Terian (nicht in CPG)] Philon d'Alexandrie, Les œuvres. Bd. 34C: Quaestiones et solutiones in Exodum I et II e versione armeniaca et fragmenta graeca. Introduction, traduction et notes Terian, Abraham, Paris 1992

[Ph., quis rer. div. her. = Philo Judaeus, Quis rerum divinarum heres sit ed. Wendland (nicht in CPG)] Philonis Alexandrini Opera quae supersunt vol. III ed. Wendland, Paul, Berolini $1962=1898$, S. 1-71

[Ph., spec. leg. = Philo Judaeus, de specialibus legibus libri I-IV ed. Cohn (nicht in CPG)] Philonis Alexandrini Opera quae supersunt vol. V ed. Cohn, Leopoldus, Berolini $1962=1906$, S. 1-265

[Ph., vita M. = Philo Judaeus, De vita Mosis ed. Cohn (nicht in CPG)] Philonis Alexandrini Opera quae supersunt vol. IV ed. Cohn, Leopoldus, Berolini $1962=$ 1902, S. 119-268

[Phot., cod. = Photius Constantinopolitanus, bibliothecae codices ed. Henry (nicht in CPG)] Photius, Bibliothèque. Tome III. Codices 186222, texte établi et traduit par Henry, René. Deuxième tirage (CUFr), Paris ${ }^{2} 2003$

[Plut., mor. = Plutarchus, Moralia ed. Sieveking] Plutarchi Moralia. Vol. II recensuerung et emendaverunt Nachstaedt, W(ilhelm) / Sieveking, W(ilhelm) / Titchener, J(ohn) B. (BT), Leipzig 1971

[Proc. G., Cant. = Procopius Gazaeus, Catena in Canticum Canticorum ed. Auwers/Guérard (CPG 7431)] Auwers, Jean-Marie [Ed.], Procopii Gazaei Epitome in Canticvm canticorvm cum praefatione a Auwers, Jean-Marie / Guérard, Marie-Gabrielle curata (CChr.SG 67), Turnhout 2011 
[Proc. G., decl. I-III = Procopius Gazaeus, Declamationes (CPG 7436) ed. Amato] Procope de Gaza, Discours et fragments. Texte établi, introduit et commenté par Amato, Eugenio, avec la collaboration de Corcella, Aldo / Ventrella, Gianluca. Traduit par Maréchaux, Pierre, Paris 2014, S. $1-116$

[Proc. G., decl. IV = Procopius Gazaeus, Declamationes (CPG 7436) ed. Corcella] Procope de Gaza, Discours et fragments. Texte établi, introduit et commenté par Amato, Eugenio, avec la collaboration de Corcella, Aldo / Ventrella, Gianluca. Traduit par Maréchaux, Pierre, Paris 2014, S. 331-344

[Proc. G., Eccl. = Procopius Gazaeus, Catena in Ecclesiasten (fragmenta) ed. Leanza (CPG 7433)] Procopii Gazaei Catena in Ecclesiasten necnon Psevdochrysostomi Commentarivs in evndem Ecclesiasten, ed. Leanza, Sandro (CChr.SG 4), Turnhout / Leuven 1978

[Proc. G., Eccl. = Procopius Gazaeus, Catena in Ecclesiasten ed. Leanza, Nachtrag (CPG 7433)] Leanza, Sandro, Un nuovo testimone della Catena sull'Ecclesiaste di Procopio di Gaza, il cod. Vindob. theol. Gr. 147 (CChr.SG 4 Suppl.), Turnhout / Leuven 1983

[Proc. G., horol. = Procopius Gazaeus, horologium ed. Amato (CPG 7438)] Procope de Gaza, Discours et fragments. Texte établi, introduit et commenté par Amato, Eugenio, avec la collaboration de Corcella, Aldo / Ventrella, Gianluca. Traduit par Maréchaux, Pierre, Paris 2014, S. $117-156$

[Proc. G. ed. Amato/Corcella/Ventrella] Procope de Gaza, Discours et fragments. Texte établi, introduit et commenté par Amato, Eugenio, avec la collaboration de Corcella, Aldo / Ventrella, Gianluca. Traduit par Maréchaux, Pierre, Paris 2014

[Proc. G., Ep. = Procopius Gazaeus, epistula ed. Maltese (CPG 7435)] Maltese, E(nrico) V(aldo) (Ed.), Un'epistola inedita di Procopio di Gaza, in: La Parola del Passato 39 (1984), S. 53-55

[Proc. G., epith. = Procopius Gazaeus, Epithalamius ed. Amato (nicht in CPG)] Procope de Gaza, Discours et fragments. Texte établi, introduit et commenté par Amato, Eugenio, avec la collaboration de Corcella, Aldo / Ventrella, Gianluca. Traduit par Maréchaux, Pierre, Paris 2014, S. $345-445$

[Proc. G., Epp. = Procopius Gazaeus, Epistulae ed. Garzya/Loenertz (CPG 7435)] Procopii Gazaei Epistulae et declamationes, ed. Garzya, Antonius / Loenertz, Raymundus-J. (SPB 9), Ettal 1963, S. 1-80

[Proc. G., epp. ad Meg. = Procopius Gazaeus, Procopii et Megethii rhetoris epistulae mutuae sex ed. Amato (nicht in CPG)] Procopius Gazaeus, Opuscula rhetorica et oratoria. Omnia primum collegit edidit apparatu 
critico instruxit Amato, Eugenio, adiuvante Ventrella, Gianluca. Cum testimoniis et fragmentis (quorum ineditum unum ex Refutatione Procli institutionis theologicae). Accedunt Procopii et Megethii rhetoris epistulae mutuae sex (BiTeu), Berolini / Novi Eboraci 2009, S. 123-134

[Proc. G., Ex. = Procopius Gazensis, Eclogarum in libros historicos Veteris Testamenti epitome. Commentarius in Exodum partim ed. Migne (CPG 7430 und CPG C 3)] PG 87/1, Sp. 512-690

[Proc. G., Gen. = Procopius Gazaeus, Eclogarum in libros historicos Veteris Testamenti epitome. Commentarius in Genesim ed. Metzler (CPG 7430 und CPG C 3)] Prokop von Gaza, Eclogarum in libros historicos Veteris testamenti epitome. Teil 1: Der Genesiskommentar, hg. Metzler, Karin (GCS NF 22), Berlin / München / Boston 2015

[Proc. G., Gen. = Procopius Gazaeus, Eclogarum in libros historicos Veteris Testamenti epitome. Commentarius in Genesim ed. Migne (CPG 7430 und CPG C 3)] PG 87/1, Sp. 21-512

[Proc. G., Gen. = Procopius Gazaeus, Eclogarum in libros historicos Veteris Testamenti epitome partim ed. Ernesti (CPG 7430 und CPG C 3)] Ernesti, Io(hannes) Christianvs Gottlieb, De Procopii Gazaei Commentariis Graecis in Heptatevchvm et Canticvm ineditis commentatio / Ad virvm excellentissimvm et doctissimvm Carolvm Avgvstvm Gottlieb Keilivm professionem philosophiae extraordinariam capessentem amicorvm aliqvot gratvlandi cavsa scripsit, Lipsiae 1785

[Proc. G., Eclogarum in libros historicos Veteris Testamenti epitome übers. Klauser (CPG 7430 und CPG C 3)] Procopii Gazaei Sophistae Commentarii in Octateuchum, hoc est, in priores octo Veteris Testamenti libros, ex uetustissimis orthodoxorum patrum, aliorumque scriptorum enarrationibus in unum corpus et perpetuum interpretationis contextum accuratissime concinnati. Nunc primum ex antiquissimo graeco codice manuscripto, quem amplissimae Reip. Augustanae Bibliotheca suppedauit, in Latinum sermonem translati Conrado Clausero Tigurino interprete. Cum duplici indice, quorum alter est locorum scripturae, qui in hoc loco sunt declarati: alter verò rerum et verborum, Tiguri 1555

[Proc. G., Gen. = Procopius Gazaeus, Eclogarum in libros historicos Veteris Testamenti epitome. Commentarius in Genesim übers. Metzler (CPG 7430 und CPG C 3)] Prokop von Gaza, Der Genesiskommentar. Aus der „Eclogarum in libros historicos Veteris Testamenti epitome“. Übersetzt und mit Anmerkungen versehen von Metzler, Karin (GCS NF 23), Berlin / Boston 2016

[Proc. G., fr. = Procopius Gazaeus, Fragmenta ed. Amato (CPG 7437)] Procope de Gaza, Discours et fragments. Texte établi, introduit et commenté par Amato, Eugenio, avec la collaboration de Corcella, Aldo / 
Ventrella, Gianluca. Traduit par Maréchaux, Pierre, Paris 2014, S. 489519

[Proc. G., imag. = Procopius Gazaeus, Descriptio imaginis ed. Amato (CPG 7437)] Procope de Gaza, Discours et fragments. Texte établi, introduit et commenté par Amato, Eugenio, avec la collaboration de Corcella, Aldo / Ventrella, Gianluca. Traduit par Maréchaux, Pierre, Paris 2014, S. $157-220$

[Proc. G., Is. = Procopius Gazaeus, Catena in Isaiam ed. Curterius (CPG 7434)] PG 87/3, Sp. 1817-2717

[Proc. G., monod. I-II = Procopius Gazaeus, Monodiae ed. Corcella (nicht in CPG)] Procope de Gaza, Discours et fragments. Texte établi, introduit et commenté par Amato, Eugenio, avec la collaboration de Corcella, Aldo / Ventrella, Gianluca. Traduit par Maréchaux, Pierre, Paris 2014, S. 447-488

[Proc. G., Pan. = Procopius Gazaeus, Panegyricus in Anastasium imperatorem ed. Amato/Ventrella (CPG 7439)] Procope de Gaza, Discours et fragments. Texte établi, introduit et commenté par Amato, Eugenio, avec la collaboration de Corcella, Aldo / Ventrella, Gianluca. Traduit par Maréchaux, Pierre, Paris 2014, S. 235-330

[Proc. G., Pan. = Procopius Gazaeus, Panegyricus in Anastasium imperatorem übers. Chauvot (CPG 7439)] Procope de Gaza, Priscien de Cesarée, Panégyrique de l'Empereur Anastase I ${ }^{\text {er }}$ Textes traduits et commentés par Chauvot, Alain (Antiquitas Reihe 1 Bd. 35), Bonn 1986

[Ps.-Athan., sabb. = Ps.-Athanasius, De sabbatis et circumcisione ed. Montfaucon (CPG 2244)] PG 28, Sp. 133-141

[Ps.-Chrys., psalm. = Iohannes Chrysostomus, In psalmos 101-107 ed. Savile (CPG 4551)] PG 55, Sp. 635-674

[Ps.-Hipp., fr. in Ps. = Hippolytus Romanus, Fragmenta in Psalmos ed. Achelis (CPG 1426 I Nota)] Achelis, Hans / Bonwetsch, G(ottlieb) Nath(anael) (Edd.), Hippolytus Werke 1/2. Exegetische und homiletische Schriften. Zweite Hälfte: Kleinere exegetische und homiletische Schriften (GCS 1 Hippolytus 1/2), Leipzig 1897, S. 136-145

[Ps.-Or., exc. in Ps. = Origenes, Libri (Tomi) in Psalmos II. Fragmenta in dinersos Psalmos in catenis. (2) PG 17, 105-114 ed. Galland (CPG 1426 II [2])] PG 17, Sp. 105-149

[Sever., poenit. = Severianus Gabbalensis, De paenitentia et compunctione ed. Montfaucon (CPG 4186)] PG 46, Sp. 323-336 (ediert unter dem Namen Chrysostomus)

[Sever., qu. ipsi subic. = Severianus Gabbalensis, in illud: Quando ipsi subiciet omnia ed. Haidacher (nicht in CPG)] Haidacher, Sebastian, 
Drei unedierte Chrysostomus-Texte einer Baseler Handschrift. II, in: ZKTh 31 (1907), S. 141-171

[Stob. = Stobaeus, Anthologia IV ed. Hense (nicht in CPG)] Ioannis Stobaei Anthologii libri duo posteriores rec. Hense, Otto. Vol. II, Berlin 1858

[SVF] Stoicorum veterum fragmenta, coll. Arnim, Ioannes ab. Bd. 2. Chrysippi fragmenta logica et physica. Editio stereotypa editionis primae (MCMIII), Stuttgart 1964 = Lipsiae / Berolini 1903

[Suppl. Hell. ed. Lloyd-Jones/Parsons] Supplementum Hellenisticum ed. Lloyd-Jones, Hugh / Parsons, Peter. Indices in hoc Supplementum necnon in Powelli Callectanea Alexandrina confecerit Nesselrath, HeinzGünther (Texte und Kommentare 11), Berolini / Novi Eboraci 1983

[Testimonien zu Proc. G.] Procopius Gazaeus, Opuscula rhetorica et oratoria. Omnia primum collegit edidit apparatu critico instruxit Amato, Eugenio, adiuvante Ventrella, Gianluca. Cum testimoniis et fragmentis (quorum ineditum unum ex Refutatione Procli institutionis theologicae). Accedunt Procopii et Megethii rhetoris epistulae mutuae sex (BiTeu), Berolini / Novi Eboraci 2009, S. 1-12

[Thdt., eran. $=$ Theodoretus, Eranistes ed. Ettlinger (CPG 6217)] Theodoret of Cyrus, Eranistes. Crit. text and prolegomena Ettlinger, Gerard H., Oxford 1975

[Thdt., Ps. 1-150 = Theodoretus, Interpretatio in Psalmos ed. Schulze (CPG 6202)] PG 80, Sp. 857-1997

[Thdt., qu. in Ex. = Theodoretus episc. Cyri, Quaestiones in Octateuchum ed. Marcos/Sáenz-Badillos (CPG 6200)] Theodoreti Cyrensis Quaestiones in octateuchum. Editio critica por Marcos, Natalio Fernández / Sáenz-Badillos, Angel (Textos y estudios "Cardenal Cisneros” 17), Madrid 1979

[Thdt., qu. in Ex. = Theodoretus episc. Cyri, Quaestiones in Octateuchum ed. Schultze (CPG 6200)] PG 80, Sp. 225-297

[Thdt., Rom.-Philm. = Theodoretus, Interpretatio in xii epistulas s. Pauli ed. Schulze (CPG 6209)] PG 82, Sp. 36-877

[Vett. Val. = Vettius Valens, Anthologiae I ed. Bara (nicht in CPG)] Vettius Valens d'Antioche, Anthologies. Livre I. Etablissement, traduction et commentaire ed. Bara, Joëlle-Frédérique (EPRO 111), Leiden u. a. 1989

[Vulgata ed. Weber/Gryson (nicht in CPG)] Biblia sacra iuxta vulgatam versionibus, adiuvantibus Fischer, B(onifatius) / Gribomont, I. [= Jean] / Sparks, H(edley) F. D. / Thiele, W(alter), recensuit et brevi apparatu critico instruxit Weber, Robertus. Editionem quartam emendatam cum sociis Fischer, B(onifatius) / Frede, H(ermann) I. [= Josef] / Sparks, H(edley) F. D. / Thiele, W(alter) praeparavit Gryson, Roger, Stuttgart ${ }^{4} 1994$ 


\section{Weitere Sekundärliteratur}

Abel, F.-M., Gaza au VI ${ }^{\mathrm{e}}$ siècle d'après le rhéteur Chorikios, in: RB 40 (1931), S. 5-31

Amato, Eugenio, Procope de Gaza, Discours et fragments. Texte établi, introduit et commenté par Amato, Eugenio, avec la collaboration de Corcella, Aldo / Ventrella, Gianluca. Traduit par Maréchaux, Pierre, Paris 2014, Introduction, S. XI-LXXXV

Amato, Eugenio (Ed.), Rose di Gaza. Gli scritti retorico-sofistici e le Epistole di Procopio di Gaza, Alessandria 2010

Amato, Eugenio, Tradition manuscrite et tradition érudite de Procope et Énée de Gaza: Deux nouvelles découvertes, in: Amato, Eugenio / Corcella, Aldo / Lauritzen, Delphine (Hgg.), L’École de Gaza. Espace littéraire et identité culturelle dans l'antiquité tardive. Actes du Colloque international de Paris, Collège de France, 23-25 mai 2013 (OLA 249), Leuven / Paris / Bristol (CT) 2017, S. 521-556

Auwers, Jean-Marie, L'interprétation du Cantique des cantiques à travers les chaînes exégétiques grecques (IPM 56), Turnhout 2011

Bammel, Caroline P(enrose) Hammond, Der Römerbrieftext des Rufin und seine Origenes-Übersetzung (AGLB 10), Freiburg 1985

Behr, John, Ireneus of Lyons. Identifying Christianity (CThC), Oxford 2013

Bischoff, Bernhard / Lapidge, Michael, Biblical Commentaries from the Canterbury School of Theodore and Hadrian, Cambridge 1994

Bitton-Ashkelony, Brouria / Kofsky, Aryeh (Hgg.), Christian Gaza in Late Antiquity (JSRC 3), Leiden / Boston 2004

Bitton-Ashkelony, Brouria / Kofsky, Aryeh, The Monastic School of Gaza (SVigChr 78), Leiden u. a. 2006

Bitton-Ashkelony, Brouria, Monasticism in Late Antique Gaza: A School or an Epoch?, in: Amato, Eugenio / Corcella, Aldo / Lauritzen, Delphine (Hgg.), L'École de Gaza. Espace littéraire et identité culturelle dans l'antiquité tardive. Actes du Colloque international de Paris, Collège de France, 23-25 mai 2013 (OLA 249), Leuven / Paris / Bristol (CT) 2017, S. $19-36$

Buchinger, Harald, Pascha bei Origenes (IThS 64) (2 Bde.), Innsbruck 2005 Buchinger, Harald, Zur Textkritik der Schrift der Origenes „Über das Pascha“, in: Palme, Bernhard (Hg.), Akten des 23. Internationalen Papyrologenkongresses Wien, 22.-28. Juli 2001 (Papyrologica Vindobonensia 1), Wien 2007, S. 61-72

Carriker, Andrew, The library of Eusebius of Caesarea (SVigChr 67),

Leiden / Boston 2003 
Cassin, Matthieu, Bulletin de patrologie, in: RSPhTh 100 (2016), S. 459-515

Ceulemans, Reinhart, A Post-Byzantine Reader of Prokopios of Gaza: Pachomios Rousanos in MS Venice, Marc. gr. II. 105 (Diktyon 70267), in: Byzantine Review 2 (2020) N01, zugänglich über: https://www.unimuenster.de/Ejournals/index.php/byzrev/article/view/2751/2625, zuletzt aufgesucht am 14. April 2020

Ceulemans, Reinhart, The Transmission, Sources and Reception of Procopius' Exegesis of Genesis: Observations in the Wake of the New Edition, in: VigChr 71 (2017), S. 205-224

Champion, Michael (W.), "The Academy and the Lyceum are Among Us": Plato and Platonism in the works of Aeneas, Zacharias and Procopius, in: Fowler, Ryan C. (Hg.), Plato in the Third Sophistic (MilleniumStudien 50), Boston / Berlin 2014, S. 259-282

Champion, Michael W., Reframing Neoplatonism in Zacharias Scholasticus' Ammonius, in: Amato, Eugenio / Corcella, Aldo / Lauritzen, Delphine (Hgg.), L'École de Gaza. Espace littéraire et identité culturelle dans l'antiquité tardive. Actes du Colloque international de Paris, Collège de France, 23-25 mai 2013 (OLA 249), Leuven / Paris / Bristol (CT) 2017, S. $99-115$

Champion, Michael W., Explaining the Cosmos. Creation and Cultural Interaction in Late-Antique Gaza (Oxford Studies in Late Antiquity), New York 2014

Clements, Ruth Anne, Peri Pascha. Passover and the Displacement of Jewish Interpretation Within Origen's Exegesis, Diss. Cambridge (MA), Harvard University 1997

[CPG I] Clavis patrum Graecorum, cvra et stvdio Geerard, Mavritii. Vol. I. Patres Antenicaeni (CChr.SG), Turnhout 1974

[CPG II] Clavis patrum Graecorum, cvra et stvdio Geerard, Mavritii. Vol. II. Ab Athanasio ad Chrysostomum (CChr.SG), Turnhout 1983

[CPG ${ }^{2} \mathrm{III}$ ] Clavis patrvm Graecorvm. Vol. III. A Cyrillo Alexandrino ad Ioannem Damascenvm, cvra et stvdio Geerard, Mavritii. Editio secunda, anastatica, addendis locupletata Noret, Jacques (CChr.SG), Turnhout 2003

[CPG $\left.{ }^{2} \mathrm{IV}\right]$ Clavis patrum Graecorum. Concilia. Catenae, cvra et stvdio Geerard, Mavritii. Deuxième édition, revue et mise à jour par Noret, Jacques (CChr.SG), Turnhout ${ }^{2} 2018$

[CPG V] Clavis patrum Graecorum. Vol. V. Indices. Initia. Concordantiae, cvra et stvdio Geerard, Mavritii / Glorie, F(rançois) (CChr.SG), Turnhout 1987 
[CPG Add.] Clavis patrum Graecorum. Vol. III A: A Cyrillo Alexandrino ad Iohannem Damascenvm. Addenda uolumini III a Noret, Jaques, parata (CChr.SG), Turnhout 2003

[CPG Suppl.] Clavis patrum Graecorum. Supplementum. Cvra et stvdio Geerard, M(avritii) / Noret, J., adivvantibus Glorie, F. / Desmet, J. (CChr.SG), Turnhout 1998

[CPL] Clavis patrum Latinorum qua in Corpus Christianorum edendum optimas quasque scriptorum recensiones a Tertulliano ad Bedam commode recludit Dekkers, Eligius opera usus qua rem praeparavit et iuvit Gaar, Memilius Vindobonensis. Editio tertia aucta et emendata (CChr.SL), Steenbrugis ${ }^{3} 1995$

Crawford, Matthew R., The Preface and Subject Matter of Cyril of Alexandria's De Adoratione, in: JThS 64 (2013), S. 154-167

Cribiore, Raffaella, The Conflict between Rhetoric and Philosophy and Zacharias' Ammonius, in: Amato, Eugenio / Corcella, Aldo / Lauritzen, Delphine (Hgg.), L’École de Gaza. Espace littéraire et identité culturelle dans l'antiquité tardive. Actes du Colloque international de Paris, Collège de France, 23-25 mai 2013 (OLA 249), Leuven / Paris / Bristol (CT) 2017, S. 73-84

de Andrés, Gregorio, Catálogo de los códices griegos desaparecidos de la Real Biblioteca de El Escorial, El Escorial 1968

Dorival, Gilles, Des commentaires de l'Écriture aux chânes, in: Mondésert, Claude (Hg.), Le monde grec ancien et la Bible (BiToTe 1), Paris 1984, S. 361-386

Dorival, Gilles, Origène dans la châne sur la Genèse, in: Adam. 23 (2017), S. 21-31

Dorival, Gilles, Rez. Prokop von Gaza. Eclogarum in libros historicos Veteris testamenti epitome. Teil 1: Der Genesiskommentar. Hg. von Karin Metzler (Die griechischen christlichen Schriftsteller der ersten Jahrhunderte NF, 22), Berlin-New York, Walter de Gruyter, 2015 [...], in: RHE 111 (2016), S. 228-233

Downey, Glanville, Gaza in the Early Sixth Century (Centers of civilization series 8), Norman (OK) 1963

Downey, Glanville, The Christian Schools of Palestine: A Chapter in Literary History, in: Harvard Library Bulletin 12 (1958), S. 297-319

Dvornik, Francis, The Embassies of Constantine-Cyril and Photius to the Arabs, in: To honor Roman Jakobson. Essays on the occasion of his 70. birthday, Bd. 1 (Janua linguarum. Studia memoriae Nicolai von Wijk dedicata, Series maior XXXI), The Hague/Paris1967, S. 569-576

Eisenhofer, Ludwig, Procopius von Gaza. Eine literarhistorische Studie, Freiburg/Brg. 1897 
Fédou Introduction = Origène, Commentaire sur l'Épître aux Romains. Texte critique établi par Bammel, C. P. Hammond. Introduction par Fédou, Michel. Traduction et notes par Brésard, Luc / Fédou, Michel. Index par Brésard, Luc (SC 543), Paris 2011

Fischer, Alexander Achilles, Der Text des Alten Testaments. Neubearbeitung der Einführung in die Biblia Hebraica von Würthwein, Ernst, Stuttgart 2009

Fuchs, Friedrich, Die höheren Schulen von Konstantinopel im Mittelalter (Byzantinisches Archiv 8), Leipzig 1926

Goodspeed, Edgar J. / Sprengling, Martin, A Descriptive Catalogue of Manuscripts in the Libraries of the University of Chicago, Chicago (IL) 1912

Grabbe, Lester L., Etymology in Early Jewish Interpretation. The Hebrew Names in Philo (Brown Judaic Studies 115), Atlanta (GA) 1988

Grafton, Anthony / Williams, Megan, Christianity and the Transformation of the Book. Origen, Eusebius, and the Library of Caesarea, Cambridge (MA) / London 2006

Haar Romeny, B(as) ter, Early Antiochene Commentaries on Exodus, in: Livingstone, E(lizabeth) A(nne) (Hg.), Papers Presented to the Twelfth International Conference on Patristic Studies Held in Oxford 1995. Biblica et Apocrypha, Ascetica, Liturgica (Studia Patristica 30), Leuven 1997, S. 114-119

Haar Romeny, Bas ter, Procopius of Gaza and His Library, in: Amirav, Hagit / Haar Romeny, Bas ter (Hgg.), From Rome to Constantinople. Studies in Honour of Averil Cameron (Late Antique History and Religion), Leuven / Paris / Dudley, MA: Peeters 2007, S. 173-190

Harnack, Adolf (von), Die Chronologie der altchristlichen Litteratur bis Eusebius. Theil 2: Die Chronologie. 2. Bd.: Die Chronologie der Litteratur von Irenaeus bis Eusebius, Leipzig 1904

Heimgartner, Martin, Pseudojustin - Über die Auferstehung. Text und Studie (PTS 54), Berlin / New York 2001

Hemmerdinger, Bertrand, Les «Notice et extraits » des bibliothèques Grecques de Bagdad par Photius, in: REG 69 (1956), S. 101-103

Henry Introduction = Photius, Bibliothèque. Tome I. Codices 1-83. Texte établi et traduit par Henry, René. Deuxième tirage (CUFr), Paris ${ }^{22003,}$ S. I-LII

Horst, Pieter W. van der, Rez. Karin Metzler (ed.), Prokop von Gaza Eclogarum in libros historicos Veteris testamenti epitome, Teil 1: Der Genesiskommentar, Die griechischen christlichen Schriftsteller der ersten Jahrhunderte NF 22. Berlin: De Gruyter, 2015. Pp. clxv +490. Hardcover. ISBN 9783110408720. €129.95, in: BMCR 2015.06.31, 
http://bmcr.brynmawr.edu/2015/2015-06-31.html, zuletzt besucht am 16. Juli 2019

Jaumann, Herbert, Critica. Untersuchungen zur Geschichte der Literaturkritik zwischen Quintilian und Thomasius (BSIH), Leiden / New York / Köln 1995

Junod, Eric, Basile de Césarée et Grégoire de Nazianze sont-il les compilateurs de la Philocalie de Origène? Réexamen de la Lettre 115 de Grégoire, in: Mémorial Dom Jean Gribomont (1920-1986) (Studia Ephemeridis „Augustinianum“ 27), Roma 1988, S. 349-360

Junod, Éric, Que savon-nous des «Scholies» ( $\mathrm{XO} \wedge \mathrm{IA}-\Sigma \mathrm{HMEI} \Omega \Sigma \mathrm{EI} \Sigma$ ) d'Origène, in: Dorival, Gilles / Le Boulluec, Alain (Hgg.), Origeniana Sexta. Origène et la bible / Origen and the Bible. Actes du Colloquium Origenianum Sextum. Chantilly, 30 août - 3 septembre 1993 (BEThL 118), Leuven 1995, S. 133-149

Karo, Georgius / Lietzmann, Iohannes, Catenarum Graecarum catalogus, in: NAWG.PH 1902, Göttingen 1902, S. 1-66, 299-350, 559-620 [In Octateuchum S. 2-17, Typ I S. 3-5, Typ II S. 5-7, Typ III S. 7-17]

Lauritzen, Delphine, De l'importance de la poésie pour penser l'école de Gaza, in: Amato, Eugenio / Corcella, Aldo / Lauritzen, Delphine (Hgg.), L'École de Gaza. Espace littéraire et identité culturelle dans l'antiquité tardive. Actes du Colloque international de Paris, Collège de France, 23-25 mai 2013 (OLA 249), Leuven / Paris / Bristol (CT) 2017, S. $37-51$

Layton, Richard A., Moses the pedagogue. Procopius, Philo, and Didymus on the Pedagogy of the Creation Account, in: Jenott, Lance / Gribetz, Sarit Kattan (Hgg.), Jewish and Christian Cosmogony in Late Antiquity (TSAJ 155), Tübingen 2013, S. 167-192

Leanza, Sandro, La catena all' Ecclesiaste di Procopio di Gaza del cod. Marc. gr. 22 (ff. $67^{\mathrm{v}}-83^{\mathrm{r}}$ ), in: Treu, Kurt (Hg.), Studia codicologica. In Zusammenarbeit mit Dummer, Jürgen / Irmscher, Johannes / Paschke, Franz (TU 124), Berlin 1977, S. 279-289

Litsas, Fotius K., Choricius of Gaza: An Approach to His Work. Introduction, Translation and Commentary, Diss. Chicago (IL) 1980

Losacco, Margherita, Ancora su testimonio della Biblioteca Foziana: sulle mani del Marc. gr. 451, in: Segno e testo 12 (2014), S. 223-255

Macé, Caroline, Animals in Pseudo-Eustathius of Antioch's Chronicle, in: Böttrich, Christfried / Fahl, Dieter / Fahl, Sabine, Von der Historienbibel zur Weltchronik. Studien zur Paleja-Literatur. Beiträge eines interdisziplinären Symposiums vom September 2017 im Alfried Krupp Wissenschaftskolleg Greifswald (GThF 31) Leipzig, angekündigt für 2020 
Makris, Georgios / Goeke-Mayr, Elizabeth, Dating the Codex Patmiacus 171: Iconoclastic Remarks on the Byzantine Illuminated Manuscripts of the Book of Job and on the Supposed Origins of the Catenas in the 6th Century, in: Brockmann, Christian / Deckers, Daniel / Harlfinger, Dieter / Valente, Stefano (Hgg.), Griechisch-byzantinische Handschriftenforschung. Traditionen, Entwicklungen, neue Wege, Berlin / Boston 2020, S. 437-459 (Text) und 815-820 (Tafeln) (im Druck)

Markschies, Christoph, Kaiserzeitliche christliche Theologie und ihre Institutionen. Prolegomena zu einer Geschichte der antiken christlichen Theologie, Tübingen 2007

Markschies, Christoph, Scholien bei Origenes und in der zeitgenössischen wissenschaftlichen Kommentierung, in: Origeniana decima. Origen As Writer. Papers of the 10th International Origen Congress, University School of Philosophy and Education "Ignatianum", Kraków, Poland. 31 August - 4 September 2009, ed. Kaczmarek, Sylwia / Pietras, Henryk, in collaboration with Dziadowiec, Andrej, Leuven / Paris / Walpole (MA) 2011, S. 147-167

McGuckin, J(ohn Anthony), Caesarea Maritima as Origen Knew It, in: Daly, Robert J. (Hg.), Origeniana Quinta: Historica, Text and Method, Biblica, Philosophica, Theologica, Origenism and Later Developments. Papers of the 5th International Origen Congress, Boston College, 14-18 August 1989 (BEThL 105), Leuven 1992, S. 3-25

Metzler, Karin, Welchen Bibeltext benutzte Athanasius im Exil? Zur Herkunft der Bibelzitate in den Arianerreden im Vergleich zur ep. ad epp. Aeg. (ANRhAW Bd. 96), Opladen 1997

Metzler, Karin, The Commentary on Exodus of Procopius of Gaza as a Source of New Fragments of Didymus the Blind, erscheint in: Amirav, Hagit / Fiori, Emiliano / Markschies, Christoph (Hgg.), The Systematization of Knowledge in Late Antiquity: Catenae, Florilegiae and Related Collections, LAHR 19, Leuven 2020

Metzler, Karin, Origenes, 'Scholia' zum Buch Genesis. Fragen der Edition und der Gattungsbestimmung, in: Adamantius 23 (2017), S. 32-44

Meyer, Martin A., History of the City of Gaza from the Earliest Times to the Present Day, New York 1907

Mioni, Elpidius, Bibliothecae Divi Marci Venetiarum Codices graeci manuscripti. Volumen I: Codices in classes a prima usque ad quintam inclusi. Pars prior: Classis I - classis II, codd. 1-120, Roma 1967

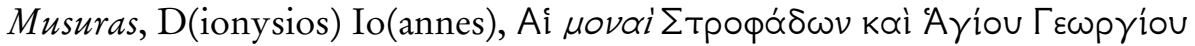

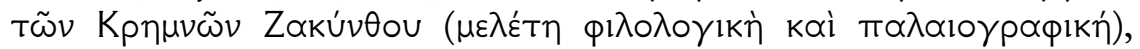
Athenai 2003 
Nelson, Anne Browning, The Classroom of Didymus the Blind, Diss. Michigan 1995

Odorico, Paolo, Dans le cahier des chroniqueurs. Le cas d'Eustathe d'Antioche, in: Signes Codoñer, Juan / Pérez Martín, Immaculada, Textual Transmission in Byzantium. Between Textual Criticism and Quellenforschung (Lectio 2), Turnhout 2014, S. 373-389

Petit, F(rançoise), La chaîne greque sur l'Exode: Description générale et problèmes spécifiques, in: Livingstone, E. A. (Hg.), Papers Presented to the Twelfth International Conference on Patristic Studies Held in Oxford 1995. Biblica et Apocrypha, Ascetica, Liturgica (Studia Patristica 30), Leuven 1997, S. 97-101

Petit, Françoise, Diodore de Tarse dans la tradition caténique sur la Genèse et l'Exode. Une mise au point, in: Muséon 112 (1999), S. 363-379

Risch, Franz Xaver, Das Handbuch des Origenes zu den Psalmen. Zur Bedeutung der zweiten Randkatene im Codex Vindobonensis theologicus graecus 8, in: Adam. 20 (2014), S. 36-48

Rokeah, David, A new onomasticon fragment from Oxyrhynchus and Philo's etymologies, in: JThS 19 (1968), S. 70-82

Runia, D(avid) T., Caesarea Maritima and the Survival of HellenisticJewish Literature, in: Raban, Avner / Holum, Kenneth (Hgg.), Caesarea Maritima. A retrospective after two millennia (DMOA 21), Leiden / New York / Köln 1996, S. 476-495

Runia, David T., Philo in Early Christian Literature: A Survey (CRINT Section III 3), Van Goreum / Assen 1993

Runia, David, Rez. Karin Metzler (ed.), Prokop von Gaza Eclogarum in libros historicos Veteris testamenti epitome. Teil 1: Der Genesiskommentar, Die griechischen christlichen Schriftsteller der ersten Jahrhunderte N.F. 22. Berlin: De Gruyter, 2015 [...]. Karin Metzler, Prokop von Gaza Der Genesiskommentar. Aus den „Eclogarum in libros historicos Veteris Testamenti epitome“ übersetzt und mit Anmerkungen versehen, Die griechischen christlichen Schriftsteller der ersten Jahrhunderte N.F. 23), Berlin: De Gruyter, 2016 [...], in: SPhiloA 29 (2017), S. 260-264

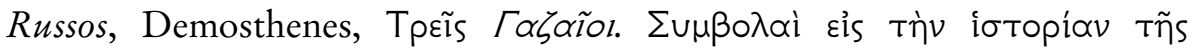

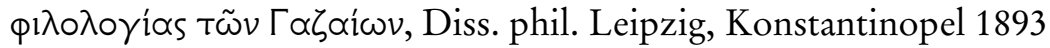

Seitz, Kilian, Die Schule von Gaza. Eine literargeschichtliche Untersuchung, Diss. phil. Heidelberg, Heidelberg 1892

Saliou, Catherine (Hg.), Gaza dans l'Antiquité Tardive. Archéologie, rhétorique et histoire. Actes du colloque international de Poitiers (6 - 7 mai 2004). Avec une préface de Flusin, Bernard, Salerno 2005, S. 171195 
Schmiele, Walter, William Shakespeare: Romeo und Julia. Vollständiger Text der Tragödie. Dokumentation, Ffm. 1963

Sivan, Hagith, Palestine in Late Antiquity, Oxford / New York 2008

Skeb, Matthias, Exegese und Lebensform. Die Proömien der antiken griechischen Bibelkommentare (Clavis commentariorum antiquitatis et medii aevi 5), Leiden / Boston 2007

Stefaniw, Blossom, Mind, Text, and Commentary. Noetic Exegesis in Origen of Alexandria, Didymus the Blind, and Evagrius Ponticus (Early Christianity in the Context of Antiquity 6), Ffm. 2010

Thévenet, Lucie, L'Ekphrasis eikonos de Procope de Gaza: Visite guidée d'une tragédie, in: Amato, Eugenio / Corcella, Aldo / Lauritzen, Delphine (Hgg.), L'École de Gaza. Espace littéraire et identité culturelle dans l'antiquité tardive. Actes du Colloque international de Paris, Collège de France, 23-25 mai 2013 (OLA 249), Leuven / Paris / Bristol (CT) 2017, S. 225-265

Tilly, Michael, Einführung in die Septuaginta, Darmstadt 2005

Tovar, A., Catalogvs codicvm Graecorvm Vniversitatis Salamantinae. I. Collectio Vniversitatis Antiqua (Acta Salmanticensia. Filosofía y Letras XV 4), Salamanca 1963

Turner, C(uthbert) H(amilton), «Two Notes on the Philocalia», in: ZNW 12 (1911), S. 231-234

Uthemann, Karl-Heinz, Was verraten Katenen über die Exegese ihrer Zeit? Ein Beitrag zur Geschichte der Exegese in Byzanz, in: Schöllgen, Georg / Scholten, Clemens (Hgg.), Stimuli. Exegese und ihre Hermeneutik in Antike und Christentum. Festschrift für Ernst Dassmann (JAC.E 23), Münster 1996, S. 287-296

Ventrella, Gianluca, Un éloge pour les Vicennalia d'Anastase Ir?, in: Amato, Eugenio / Corcella, Aldo / Lauritzen, Delphine (Hgg.), L'École de Gaza. Espace littéraire et identité culturelle dans l'antiquité tardive. Actes du Colloque international de Paris, Collège de France, 23-25 mai 2013 (OLA 249), Leuven / Paris / Bristol (CT) 2017, S. 209-223

Villani, Barbara, Some Remarks on the Textual Tradition and the Literary Genre of Cyril of Alexandria's De adoratione et cultu in spiritu et veritate, in: Vinzent, Markus (Hg.), Papers Presented at the Seventeenth International Conference on Patristic Studies held in Oxford 2015. The Second Half of the Forth Century. From the Fifth Century Onwards (Greek Writers). Gregory Palamas’ Epistula III (Studia Patristica 96), Leuven / Paris / Bristol (CT) 2017, S. 214-223

Wacht, Manfred, Aeneas von Gaza als Apologet. Seine Kosmologie im Verhältnis zum Platonismus (Theoph. 21), Bonn 1969 
Westberg, David, Celebrating with Words. Studies in the Rhetorical Works of the School of Gaza, Diss. Uppsala 2010

Westberg, David, Rhetorical Exegesis in Procopius of Gaza's Commentary on Genesis, in: Vinzent, Markus (Hg.), Studia Patristica LV. Papers presented at the Sixteenth International Conference on Patristic Studies held in Oxford 2011. Vol. 3: Early Monasticism and Classical Paideia, Leuven / Paris / Walpole (MA) 2013, S. 95-108

Witte, Bernd, Die Schrift des Origenes „Über das Pascha“. Textausgabe und Kommentar (Arbeiten zum spätantiken und koptischen Ägypten 4), Altenberge 1993

Wutz, Franz, Onomastica sacra. Untersuchungen zum liber interpretationis nominum Hebraicorum des Hl. Hieronymus (TU 41) (2 Bde.), Leipzig 1914-1915 